\title{
Herdabilidade de características de crescimento em bovinos da raça Nelore utilizando métodos da Máxima Verossimilhança Restrita e Inferência Bayesiana
}

\author{
Evangelista, A.F. ${ }^{1} ;$; Cavalcante, D.H. ${ }^{2}$; Fonseca, W.J.L. ${ }^{3}$; Barros Junior, C.P. ${ }^{4}$; Campelo, J.E.G. ${ }^{2}$ e Sousa Júnior, S.C. ${ }^{2}$
}

'Programa de Pós-Graduação em Zootecnia, Universidade Federal do Paraná, Curitiba, Brasil.

2Programa de Pós Graduação em Ciência Animal , Universidade Federal do Piauí, Teresina, Brasil.

3Programa de Pós-Graduação em Zootecnia, Universidade Estadual do Sudoeste da Bahia, Itapetinga, Bahia, Brasil.

4Programa de Pós-Graduação em Zootecnia, Universidade Estadual do Oeste do Paraná, Marechal Cândido Rondon, Paraná, Brasil.

\section{PalaVRas ChaVe ADICIONAIS}

Avaliação Genética.

Bos indicus.

Parâmetros genéticos.

Carne.

\section{RESUMO}

O melhoramento animal é uma ciência preditiva que tem na variabilidade genética do rebanho a sua base e na seleção uma importante ferramenta de ação, cuja eficiência é ponderada pela herdabilidade das características de importância econômica, que é um parâmetro específico de cada rebanho. Por ser bom indicador das consequências do manejo genético nos sistemas de produção, a herdabilidade rece beu atenção da pesquisa e contribuição da evolução de recursos computacionais e de métodos estatísticos para a estimação de seus componentes de variância, resultando em estimativas com maior precisão, inicialmente pelo método de mínimos quadrados, em seguida por máxima verossimilhança e estatísticas bayesianas. O rebanho Nelore do Brasil passou por essas etapas no seu processo de melhoramento genético, que tem sido norteado pela seleção em características de crescimento (pesos em idades padrão) como fenótipos de interesse econômico, que apresentam herdabilidade de moderada a alta magnitude. A herdabilidade tem sido estimada por diversos procedimentos, mas a estimação de componentes de variância por máxima verossimilhança restrita (REML) tem sido preferida. No entanto, limitações como a demanda computacional e a grande quantidade de dados tem favorecido o uso da Inferência Bayesiana. Partindo dessas premissas, o objetivo com esta revisão é abordar a herdabilidade das características de crescimento em bovinos da raça Nelore estimada pelos métodos de Máxima verossimilhança restrita e Inferência Bayesiana.

\section{Heritability of growth traits in Nellore cattle using Restricted Maximum Likelihood and Bayesian inference methods}

\section{SUMMARY}

\section{ADDITIONAL KEYWORDS}

Genetic evaluation.

Bos indicus.

Genetic parameters.

Beef.

\section{INFORMATION}

\section{Cronología del artículo.}

Recibido/Received: 19.03.2018

Aceptado/Accepted: 27.04 .2019

On-line: 15.07.2019

Correspondencia a los autores/Contact e-mail:

amaurifelipe17@hotmail.com
Animal breeding is a predictive science which is carried out on the basis of the genetic variability in the herd and uses selection as an important tool, whose efficiency is assessed by the heritability of the traits of interest, which is a specific parameter of each herd. Due to its good ability to indicate the consequences of the genetic management in production systems, heritability has attracted attention of researchers and received contribution of the evolution of computational resources and statistical methods for the estimation of variance components. This has resulted in higher precision estimates, initially by the Least Squares Method, later by Maximum Likelihood and Bayesian statistics. The Brazilian Nellore herd has undergone these stages in its genetic improvement process, which has been guided by the selection of growth traits (weights at standard ages) as phenotypes of economic interest, which showed moderate to high heritability. Heritability has been estimated by different approaches, but the estimation of variance components by Restricted Maximum Likelihood (REML) has been the favorite. However, limitations such as computational demand and big amount of data have favored the use of Bayesian Inference. Based on these assumptions, the objective of this review is to discuss heritability of growth traits in Nellore cattle, using Maximum Likelihood and Bayesian Inference.

\section{INTRODUÇÃO}

A magnitude de ganhos genéticos em um sistema de produção depende de diversos fatores. Um deles é a eficiência na identificação dos animais com maior valor genético aditivo nas características ponderais a serem consideradas no critério de seleção. Pequena quantidade e má utilização dos dados disponíveis, 
associado a modelos estatísticos e metodologias impróprias, diminui acurácia da avaliação genética (Valente et al., 2008, p. 390).

As características de crescimento são relevantes para aumentar a eficiência econômica de sistema de produção de bovinos de corte, destacando-se como critério de seleção com potencial para proporcionar elevado ganho genético por geração. Assim, com o progresso da pecuária no país e a obrigação de melhorar o rebanho para ser competitivo comercialmente, o pecuarista tem cada vez mais procurado novos meios para favorecer as características de importância econômica (Lira et al., 2008, p.2).

Nesse processo, a qualidade da estimativa dos parâmetros genéticos como a herdabilidade das características, é importante por ser uma das ferramentas utilizadas no processo de seleção e para a predição do valor genético dos animais. A utilização desse parâmetro como informação, ajuda na tomada de decisão ao realizar a seleção no rebanho, principalmente em bovinos Nelore, que, segundo o IBGE (2016), é a raça de corte com maior contingente no rebanho nacional.

Os pesos corporais mensurados ao longo da vida dos animais tem sido as principais fontes de informação para a avaliação genética de gado de corte, favorecido pela facilidade de mensuração (Araujo Neto et al., 2011, p.3128), alem de apresentar herdabilidade de moderada a alta magnitude (Ferreira et al., 2017, p.1090). São dados que podem ser classificados como longitudinais medidos diversas vezes durante a vida do animal, e diversos modelos com base nas equações de modelos mistos têm sido utilizados para avaliação genética de características desta natureza, com a obtenção de melhores preditores lineares não viesados (BLUP) correspondentes aos valores genéticos dos animais avaliados.

Entretanto, independente do modelo, os métodos de estimação de componentes de variância e parâmetros genéticos mais utilizados emanimais são da máxima verossimilhança restrita (REML) e Inferência Bayesiana. O método da máxima verossimilhança restrita foi introduzido no melhoramento animal por Patterson e Thompson (Carneiro Junior et al., 2004, p. 331), e tem sido um dos métodos mais utilizado pelos pesquisadores.

Embora a metodologia REML seja considerada padrão nas avaliações genética dos animais, a Inferência Bayesiana, aparece como uma alternativa e vem ganhando espaço em pesquisas cientificas, Faria et al. (2007, p. 75) podendo ser utilizada tanto em pequenos ou grandes conjuntos de dados. No Brasil, ambos os métodos, tem sido aplicado em estudos com dados de bovinos da raça Nelore. Sendo assim, o objetivo com esta revisão é abordar a herdabilidade das características de crescimento em bovinos da raça Nelore estimada por métodos da Máxima verossimilhança restrita e Inferência Bayesiana.

\section{PECUÁRIA DE CORTE NO BRASIL}

A pecuária de corte no Brasil é uma atividade antiga, praticamente teve inicio no período da colonização
(Santos et al., 2014). O grande consumo de carne bovina exigida pelos mercados internos e externos tem feito com que a pecuária de corte brasileira produzisse carnes em grande quantidade e de boa qualidade. Assim a cadeia produtiva da carne bovina brasileira vem se fortalecendo nos últimos anos e contribuindo para colocar o agronegócio em posição de destaque na economia internacional.

Entretanto, a posição do Brasil no cenário mundial não está consolidada e, para que isto ocorra, há necessidade de se promover melhorias contínuas no rebanho de bovinos (Santos, G. et al., 2012, p.97). Em 2012, a produção nacional de carne foi de 9,2 milhões de toneladas, representando aproximadamente $16 \%$ de toda produção mundial (Pinto et al., 2015, p.203), no ano de 2015 o rebanho mundial tinha um total de 971.482 .600 cabeçasde bovinos, o Brasil ocupava o segundo maior rebanho do mundo,com 215,2 milhões de cabeças, representando aproximadamente $22,2 \%$ de todo rebanho mundial, ficando apenas atrás dos Índia (IBGE, 2016).

No cenário brasileiro, a região Centro-Oeste aparece como uma grande produtora de carne bovina, com um efetivo que é responsável por 33,5\% do gado bovino nacional (IBGE, 2016). Carvalho et al. (2017, p.85) relatam que a principal característica no desenvolvimento da pecuária de corte no Brasil é a heterogeneidade nos sistemas de produção e nos mecanismos de gestão e de comercialização do gado.

Além disso, outro fator determinante para colocar o Brasil em posição de destaque como grande produtor na bovinocultura de corte, tem sido a intensificação do uso do melhoramento genético como ferramenta para o aumento da eficiência da produção animal (Marques et al., 2013, p.159). Para isto são utilizadas ferramentas, como, cruzamento e seleção, Silva et al. (2015, p.279) no qual existem varias publicações atestando o seu beneficio no Brasil, principalmente nas características de crescimento.

Na bovinocultura de corte, é importante selecionar animais tendo em vista melhorias nos desempenhos produtivos e reprodutivos, pois esses animais apontam a eficiência total de produção, tanto do ponto de vista genético como econômico (Boligon et al., 2008, p.256). Assim tornando a pecuária de corte no Brasil cada vez mais competitiva, fazendo-se necessários aumentos constantes de produtividade de carne bovina.

\section{Raça Nelore}

O Brasil apresenta o maior rebanho comercial de bovinos do mundo, com maioria composta por animais zebuínos (Bos taurus indicus), dentre os quais se destaca a raça Nelore, que é uma Raça bovina proveniente da Índia, chegou ao Brasil por volta do século XVIII, e se tornou predominante no rebanho brasileiro. Alastrouse por toda a América do Sul e, principalmente no Brasil devido à sua alta fertilidade a pasto e capacidade de sobrevivência.

No Brasil há duas variedades de bovinos da raça Nelore, o Nelore padrão e os animais da raça Nelore variedade Mocha (Nelore Mocho), estes dois grupos genéticos compõem os maiores efetivos de zebuínos criados nas regiões do Brasil, e apresentam diferenças 
além da presença ou não de chifres, portanto são populações muito diferentes (Ambrosini et al., 2016, p.178).

E nessas ultimas décadas, tem-se aumentado a utilização da Raça Nelore em programas de melhoramento animal, e hoje é a raça base para o cruzamento de gado de corte no Brasil. Isso se deve as importações na década de 1960, quando foram introduzidas de alguns touros importantes devido as suas características fenotípicas e que contribuíram para a caracterização, conformação e melhoramento do rebanho Nelore nacional (História, 2011).

A busca dos criadores por animais melhores e mais eficientes incentivou o surgimento de programas de melhoramento animal, estes programas têm promovido importantes avanços na raça Nelore ao permitir a aceleração do progresso genético e maior difusão do material genético superior (Santana \& Josahkian, 2011, p.1). Dentro deste enfoque de melhoristas, estudos em parâmetros genéticos passaram a ter atenção especial.

A relevância desta raça dentro da pecuária de corte nacional é indicada pela expressiva venda de sêmen no ano de 2012, atingindo 263.134 doses vendidas, que correspondeu a um aumento de 1,65\% em relação ao ano de 2011, é de 27,46\% a mais que em 2010 (Amaral et al., 2014). E as vantagens destes animais no País são conhecidas e podem ser sumariadas pela capacidade de produção em sistemas com baixo nível e má distribuição da oferta de nutrientes, sob condições climáticas e sanitárias adversas (Santos, N. et al., 2012, p.2).

\section{CaracterísticAs de CRESCIMENTO}

Na pecuária de corte, os criadores estão cada vez mais preocupados com o crescimento animal, visto que o crescimento rápido é desejável nos bovinos de corte, sendo que animais que apresentam maiores ganhos de peso necessitam de menos dias para serem abatidos. Dessa forma o melhoramento genético de bovinos de corte no Brasil tem sido conduzido, principalmente pela seleção com base nos pesos corporal padronizados em diferentes idades, ganhos de peso e número de dias para atingir determinado peso (Ferriani et al., 2013, p.124).

A melhoria do potencial genético de uma raça para produção de carne pode ser alcançada por meio de avaliações genéticas dos animais em características relacionadas com o crescimento e posterior seleção dos indivíduos geneticamente superiores. As principais informações para avaliação genética são os pesos mensurados durante o período de crescimento

O peso ao nascer, na desmama, ao ano e sobreano, são características de crescimento que formam a base principal das informações utilizadas em avaliações genéticas na pecuária de corte, e o motivo de utilizar as características de crescimento como critério de seleção nos programas de melhoramento genético de bovinos de corte, é o fato de elas serem de fácil mensuração e apresentarem herdabilidades com valores moderados, o que propicia ganhos genéticos razoáveis ao longo das gerações e por estarem diretamente relacionadas à quantidade produzida de carne (Silva et al., 2012, p.1167).

Estudos associados ao crescimento dos animais de corte são relevantes em programas de melhoramento genético, por permitir auxilio à seleção para ganho de peso e precocidade (Souza et al., 2010, p. 672). Essas características são influenciadas tanto pelos próprios genes dos animais como por fatores ambientais, o crescimento desses indivíduos na fase que antecede a desmama está intimamente ligado ao potencial de crescimento pré e pós-nascimento do bezerro, bem como à habilidade materna da vaca (Sousa Júnior et al., 2011, p. 351).

O melhoramento genético das características de crescimento em bovinos de corte é dependente da estimação acurada dos componentes de covariância, essa acurácia é influenciada pela quantidade e qualidade das informações obtidas, o modelo estatístico aplicado e o próprio método de estimação de componentes de covariância. A evolução de programas estatísticos e de recursos computacionais tem facilitado o particionamento dos componentes de covariância, possibilitado estimativas dos efeitos genéticos e ambientais mais acuradas, (Sousa et al., 2009, p.212), posteriormente melhorando a resposta à seleção

\section{MÉTODOS DE ESTIMAÇÃO DE COMPONENTES DE COVARIÂNCIA}

\section{MÁXIMA VEROSSIMILHANÇA RESTRITA - REML}

A predição dos valores genéticos e os métodos de seleção necessita das estimativas dos componentes de variância (Resende et al., 1996, p.24), onde são importantes na obtenção da estatística $\mathrm{F}$, na construção de índices de seleção, na análise de modelos mistos com vistas à predição linear do tipo BLUP, na estimativa dos parâmetros genéticos, fenotípicos e de meio ambiente, no planejamento de programas de melhoramento genético (Lôbo, 2004, p. 3). Existem vários métodos de estimação de componentes de variância, dentre os quais aparece o Método da Máxima Verossimilhança Restrita (REML).

O método REML é utilizado em dados desbalanceados e apropriado para estimação de parâmetros genéticos em dados de melhoramento animal, pois além de considerar a perda de graus de liberdade resultante da estimação dos efeitos fixos, as estimativas caem sempre dentro do espaço paramétrico (Barbosa et al., 2015, p. 62). Esse método tem essa denominação, pelo fato de cada observação ser dividida em duas partes independentes, uma se referindo aos efeitos fixos e a outra aos efeitos aleatórios.

Este método é relevante no melhoramento animal para estimar as variâncias fenotípicas e outros componentes, as razões são justificadas pelas propriedades desses estimadores. O REML possui varias características desejáveis, quando as distribuições são normais, 
elas minimizam os vieses das estimativas de variâncias e covariâncias decorrentes de acasalamento seletivo, seleção através de gerações e descartes seqüenciais (Freitas et al., 1994, p. 395).

As vantagens dessa metodologia estão na capacidade de geração de estimativas não negativas de componentes de variância, não ser afetado por mudanças nos efeitos fixos, suficiência, consistência e eficiência, é flexível não exigindo delineamentos balanceados (Resende et al., 1996, p.25), além de reduzir o viés, em decorrência da seleção, e a capacidade de utilizar toda informação disponível (Falcão et al., 2009, p. 1479).

\section{INFERÊNCIA BAYESIANA}

Estimar os componentes de variância tem sido relevante na bovinocultura de corte, mas para a obtenção dessas estimativas é preciso de um método estatístico apropriado, que se adeque aos dados queserão avaliados. Embora a metodologia de Máxima verossimilhança Restrita (REML) venha sendo usada como padrão para a avaliação genética dos animais, ela apresenta algumas limitações, com isso faz se o uso da Inferência Bayesiana, que consiste em uma abordagem estatística que trata os parâmetros populacionais como variáveis aleatórias (Nogueira et al., 2003, p. 1615).

A estatística Bayesiana foi fundamentada no teorema de Thomas Bayes, em 1761, no melhoramento genético animal, o método bayesiano foi introduzido por Gianola e Fernando (Carneiro Junior et al., 2004, p. 331). Santos, N. et al. (2012, p. 2) relatam que este método permite solucionar muitos problemas que não foram resolvidos no passado em virtude da impossibilidade deresolução de múltiplas integrais na estimação dos componentes de (co)variância.

O uso da metodologia bayesiana para estimar os componentes de variância, surgiu como uma opção de grande importância em relação aos métodos utilizados nas análises, Yokoo et al. (2013, p. 247) tendo mostrado eficiência e fácil implementação para estimação confiável de parâmetros, pois a sua aplicação em análises genéticas permite a obtenção de densidades posteriores das variáveis estudadas e pode ser utilizada tanto em pequenos ou grandes conjuntos de dados, não sendo necessário o conhecimento da distribuição inicial do parâmetro que se deseja estimar (Faria et al., 2008, p.333).

Diante disso, a Inferência Bayesiana vem ganhando espaço no melhoramento genético animal para solucionar problemas relacionados à estimação dos parâmetros genéticos, e a vantagem de usar a Inferência Bayesiana no melhoramento animal é porque ela descreve os valores dos parâmetros usando probabilidade, pois ela apresenta o nível de incerteza que se tem sobre a característica em um evento amostral. No entanto, os métodos bayesianos têm propiciado novas perspectivas a questões relacionadas à estimação de componentes de variância e avaliações genéticas (Faria et al., 2007).

\section{PARÂMETROS GENÉTICOS}

HERDABILIDADE $\left(\mathrm{H}^{2}\right)$
Os parâmetros genéticos são definidos pelos componentes de variância, nas diversas populações, ou seja, são específicos para determinada população. Apresentam um papel relevante nos programas de melhoramento genético porque fornecem as informações necessárias para definição de índices de seleção e para avaliação genética (Guimarães \& Farias 2010, pag. 8), alem disso é possível verificar se está havendo melhoramento genético no rebanho.

As estimativas dos parâmetros genéticos permitem avaliar a eficiência da estratégia do melhoramento e possibilitar que a seleção seja propicia para as características de importância econômica, além de apoiar na definição dos objetivos da seleção. Quando se objetiva estudar parâmetros genéticos, deve se utilizar material representativo da população, dentre os parâmetros podemos evidenciar a herdabilidade.

Na literatura apresenta alguns trabalhos com estimativas de parâmetros genéticos na raça Nelore e os objetivos desses estudos geralmente é conhecer o potencial genético do rebanho nacional. Portanto, tornase necessário que sejam estimados parâmetros genéticos com precisão, com os quais se estima os coeficientes de herdabilidade das características de importância econômica, que são importantes para o planejamento de programas de melhoramento genético mais eficiente (Weber, et. al., 2009, p. 833).

Para o melhoramento genético animal, a herdabilidade é um parâmetro de grande importância e mais adequado ao rebanho, pois estima a capacidade de transmitir características aos descendentes. Lira et al. (2008, p.2), é um parâmetro que mede a proporção da variância genética sobre a variância fenotípica, ou seja, é a proporção herdável da variabilidade total.

È representada com o símbolo $h^{2}$ e expressa o grau de confiança do valor fenotípico como indicador do valor genético. Para administrar um programa de melhoramento genético, é essencial ter conhecimento sobre a herdabilidades, porque alguns pareceres são tomados em função de sua magnitude. Elas são estimadas a partir de uma análise de variância e seus valores podem variar de zero (0) a unidade (1).

Esses valores indicam que tanto os fatores ambientais, quanto os fatores genéticos, estão atuando na variância fenotípica. Herdabilidade igual a unidade, significa que as diferenças fenotipicamente entre os indivíduos de uma população, são causadas pela diferença genética entre um a outro. Quando é igual a zero, significa que a variedade de características não é de origem genética, ou seja, não existe relação alguma entre o valor genético e valor fenotípico entre os indivíduos de uma população.

Estudos de herdabilidade voltada para produção animal vêm crescendo dentro do Brasil, pois o mesmo serve como auxilio em programas de melhoramento animal. E o número de trabalhos publicados contendo estimativas de herdabilidade para características de crescimento em bovinos de corte, é suficientemente grande para se conduzir análises visando determinar que fatores afetem essas características e fazer reco- 
mendações considerando valores combinados apropriados (Giannotti et al., 2002, p.440).

As características de crescimento, tais como pesos padronizados a determinadas idades têm sido as características mais comumente utilizadas em programas de seleção, principalmente, por serem características de interesse econômico e por apresentarem facilidade de obtenção, além de responderem favoravelmente aos processos de seleção impostos, uma vez que apresentam coeficientes de herdabilidade geralmente elevados (Lôbo et al., 2002, p.6). Diversos autores relatam diferentes valores deherdabilidade para as características de crescimento, Tabela I.

De modo geral, percebe-se grande variação nos valores das herdabilidades para as características de crescimento em bovinos da raça Nelore reportadas na literatura científica, o que pode ser justificado, são as diferenças ambientais no qual os animais foram submetidos, estruturas de dados diferentes com as restrições impostas, diferenças nos modelos estatísti- cos utilizados e diferenças nas metodologias adotadas pelos autores. Há poucos estudos que estima a herdabilidade nas idades iniciais, o que justifica a quantidade insuficiente de registro.

\section{CONCLUSÕES}

Estudos de parâmetros genéticos na bovinocultura de corte são uma das alternativas em programas de melhoramento para selecionar animais com grande potencial para produção de carne. Dessa forma, os parâmetros genéticos surgiram como uma alternativa pra fazer avaliações genéticas e são notórios os benefícios que os mesmos trazem aos pecuaristas, melhorando significativamente o rebanho. Sendo assim, devem-se estimular estudos que utiliza metodologias adequadas para estimar esses parâmetros acurados, pois os mesmos são essenciais para conferir melhorias e ajustes na produção. Em virtude disso, pesquisas no âmbito do melhoramento animal, voltada a utilização de metodologias aplicado na produção para estimar os

Tabela I. Estimativas de herdabilidades $\left(\mathrm{h}^{2}\right)$ do peso ao nascer (PN), aos 120 (P120), 205 (P205), 365 (P365) e 550 (P550) dias de idades em bovinos da raça Nelore de acordo com diversos autores pelos métodos da Máxima Verossimilhança Restrita e Inferência Bayesiana (Estimates of heritabilities $\left(\mathrm{h}^{2}\right)$ of birth Weight (BW), 120 (P120), 205 (P205), 365 (P365) and 550 (P550) days of age in Nellore cattle according to several authors by the methods of restricted maximum likelihood and Bayesian inference).

\begin{tabular}{|c|c|c|c|c|c|c|}
\hline \multirow[b]{2}{*}{ Autores } & \multicolumn{6}{|c|}{ Herdabilidade $\left(\mathrm{h}^{2}\right)$} \\
\hline & PN & P120 & P205 & P365 & P550 & Métodos \\
\hline Kamei et al. (2017, p.1507) & 0,17 & 0,14 & 0,17 & 0,14 & 0,21 & REML \\
\hline Barros et al. (2017, p. 4) & - & - & 0,42 & 0,54 & 0,65 & REML \\
\hline Lopes et al. $(2017$, p. 7$)$ & - & 0,28 & - & 0,49 & - & IB \\
\hline Nepomuceno et al. (2017, p. 257) & - & - & - & 0,56 & 0,59 & REML \\
\hline Barbosa et al. (2017, p. 448) & - & - & 0,15 & 0,19 & 0,26 & REML \\
\hline Pereira et al (2017, p. 470) & - & 0,28 & - & 0,28 & - & IB \\
\hline Ambrosini et al. (2016, p.181) & - & - & 0,34 & - & - & IB \\
\hline Bresolin et al. (2015, p. 198) & - & - & 0,24 & - & - & IB \\
\hline Ferreira et al. (2014, p.15) & - & - & 0,27 & 0,54 & 0,39 & REML \\
\hline Ferreira et al. (2014, p.15) & - & - & 0.33 & 0,61 & 0,41 & REML \\
\hline Pedrosa et al. (2014, p.1808) & 0,31 & - & 0,22 & - & - & REML \\
\hline Amaral et al. (2014, p.265) & - & - & 0,19 & 0,24 & 0,18 & REML \\
\hline Lacerda et al. (2014, p.13) & - & - & 0,21 & 0,21 & 0,19 & IB \\
\hline Lopes et al. (2013, p.3) & - & 0,43 & - & 0,72 & - & IB \\
\hline Santos, G et al. (2012, p.99) & - & - & 0,33 & 0,51 & 0,41 & REML \\
\hline Santos, N et al.(2012, p.4) & - & - & - & 0,23 & 0,15 & IB \\
\hline Santos, N et al. (2012, p.5) & - & - & - & 0,34 & 0,32 & IB \\
\hline Araujo Neto et al. (2011, p.3132) & - & 0,23 & - & 0,35 & 0,39 & IB \\
\hline Gonçalves et al. (2011, p.161) & - & - & 0,6 & 0,68 & 0,75 & REML \\
\hline Martínez-González et al. (2010, p.889) & 0,59 & & 0,29 & 0,24 & - & REML \\
\hline Albuquerque et al. (2008, p.242) & 0,33 & 0,15 & 0,20 & 0,33 & 0,49 & REML \\
\hline Faria et al $(2004$, p.1) & - & - & - & 0,49 & - & IB \\
\hline Holanda et al. (2004, p.189) & 0,23 & - & 0,12 & - & - & REML \\
\hline $\begin{array}{l}\text { REML = Máxima Verossimilhança Restr } \\
\text { IB= Inferência Bayesiana }\end{array}$ & & & & & & \\
\hline
\end{tabular}


parâmetros genéticos em bovinos de corte, fazem-se necessárias e devem ser desenvolvidas a fim de proporcionar animais que possam expressar o máximo de seu potencial produtivo.

\section{BIBLIOGRÁFIA}

Albuquerque, LG \& Faro, LE 2008, 'Comparações entre os valores genéticos para características de crescimento de bovinos da raça Nelore preditos com modelos de dimensão finita ou infinita', Revista Brasileira de Zootecnia, v.37, no.2, pp.238-246,

Amaral, RS, Carneiro, PLS, Martins Filho, R, Ambrosini DP \& Malhado, CHM 2014, 'Tendências, parâmetros fenotípicos e genéticos de características de crescimento em bovinos Nelore mocho do Nordeste brasileiro', Revista Brasileira de Saúde e Produção Animal, v. 15, no. 2, pp. 261-271

Ambrosini, DP, Malhado, CHM, Martins Filho, R \& Carneiro, PLS 2016 , 'Interação genótipo $x$ ambiente via modelos de normas de reação para características de crescimento em bovinos Nelore', Pesquisa Agropecuária Brasileira, v. 51, no. 2, pp. 177-186.

Araujo Neto, F R, Lôbo, RB, Mota, MDS \& Oliveira, HND 201 1, 'Genetic parameter estimates and response to selection for weight and testicular traits in Nelore cattle', Genetics and Molecular Research, v. 10, no. 4, pp. 3127-3140

Barbosa, ACB, Carneiro, PLS, Rezende, MPG, Ramos, IO, Martin-Filho, R \& Malhado, CHM 2017 'Parâmetros genéticos para características de crescimento e reprodutivas em bovinos Nelore no Brasil ', Archivos de zootecnia, v. 66, no. 255, pp. 447-450.

Barbosa, L, Lopes, PS, Carneiro, PCS, Regazzi, AJ, Torres, RA, Costa, ARC \& Santana Junior, ML 2015, 'Comparação entre modelos para estimação de parâmetros genéticos em características de desempenho em suínos da raça Large White', Revista Ceres, v. 55, no. 1, pp. 60-65.

Barros, IC, Carneiro, PLS, Mota, RR, Silva, LP, Martins Filho, R \& Malhado, CHM 2017, 'Genetic parameters estimation of growth in Polled Nellore cattle via random regression models', Livestock Research for Rural Development, v. 29, no. 12, pp. 1-6.

Boligon, AA, Albuquerque, LD \& Rorato, PRN 2008, 'Associações genéticas entre pesos e características reprodutivas em rebanhos da raça Nelore', Revista Brasileira de Zootecnia, v. 37, no. 4, pp. 596-601.

Bresolin, T, Everling, DM, Rorato, PRN, Braz, CU \& Breda, FC 2015, Influence of genotype-environment interaction on the classification of Nellore bulls in Southern Brazil', Acta Scientiarum. Animal Sciences, v. 37 , no. 2, pp. 195-201.

Carneiro Júnior, JM, Lopes, PS, Torres, RDA \& Euclydes, RF 2004, 'Avaliação de métodos de estimação de componentes de variância utilizando dados simulados', Revista Brasileira de Zootecnia, v.33, no. 2, pp.328-336.

Carvalho, TB \& Zen, S 2017, 'A cadeia de Pecuária de Corte no Brasil: evolução e tendências Revista', iPecege, v. 3, no. 1, pp. 85-99.

Falcão, AJS, Martins, EN, Costa, CN \& Mazucheli, J 2009, ‘Efeitos do número de animais na matriz de parentesco sobre as estimativas de componentes de variância para produção de leite usando os métodos de máxima verossimilhança restrita e Bayesiano', Revista Brasileira Zootecnia, v.38, no.8, pp.1478-1487.

Faria, CU, Albuquerque, LG, Reyes, A, Bezerra, LAF \& Lôbo, RB 2008, 'Estimativas de correlações genéticas entre escores visuais e características de crescimento em bovinos da raça Nelore utilizando modelos bayesianos linear-limiar', Ciência Animal Brasileira, v. 9, no. 2, pp. 327-340.

Faria, CU, Magnabosco, CDU, Reyes, ADL, Lôbo, RB \& Bezerra, LAF 2007, 'Inferência Bayesiana e sua aplicação na avaliação genética de bovinos da raça nelore: revisão bibliográfica', Ciência Animal Brasileira, v. 8, no. 1, pp. 75-86.

Faria, CU, Magnabosco, CDU, Reyes, AD, Lôbo, R, Bezerra, I, Barbosa, V \& Paula, JH 2004, 'Inferência Bayesiana para estimação de parâmetros genéticos de características de crescimento de bovinos da raça nelore', Embrapa Cerrados-Fôlder/Folheto/Cartilha (INFOTECA-E).
Ferreira, JL, Lopes, FB, Marques, EG, Silva, MC, Assis, AS, Pereira, LS \& Nepomuceno, LL 2014, 'Estudo genético quantitativo em características produtivas de bovinos da raça Nelore criados na Região Norte do Brasil', Brazilian Journal of Veterinary Medicine, v. 36, no. 1, pp. 11-17.

Ferreira, JL, Bresolin, T, Lopes, FB, Garcia, JAS, Nepomuceno, LL., Schmidt, $A B$ \& Lobo, RB 2017, 'Modelos de regressão aleatória para característica de crescimento em bovinos da raça Guzerá', Ciência Animal Brasileira, v.18, e-39566, pp.1-12.

Ferriani, L, Albuquerque, LG, Baldi, FSB, Venturini, GC, Bignardi, AB, Silva, JA \& Oliveira, JA. 2013, 'Parâmetros genéticos de características de carcaça e de crescimento de bovinos da raça Nelore', Archivos de zootecnia, v. 62, no. 237, pp. 123-129.

Freitas, AR, Favoretti, ADC \& Alencar, MM 1994, 'Uso da máxima verossimilhança restrita e transformação canônica para estimação de parâmetros genéticos de características de crescimento em bovinos', Revista da Sociedade Brasileira de Zootecnia, v. 23, no. 3, pp. 394-401.

Giannotti, JG, Packer, IU \& Mercadante, MEZ 2002, 'Meta-análise para estimativas de correlação genética entre pesos ao nascer e desmama de bovinos', Scientia Agricola, Piracicaba, v. 59, no.3, p. 435-440.

Gonçalves, FM, Pires, AV, Pereira, IG, Garcia, DA, Farah, MM, Meira, CT \& Cruz, VAR 2011, 'Avaliação genética para peso corporal em um rebanho Nelore', Arquivo Brasileiro de Medicina Veterinária e Zootecnia, v. 63, no. 1, pp. 158-164.

Guimarães, PHR \& Faria, CU 2010, 'Caracterização da raça Nelore Mocho no Brasil: Revisão bibliográfica'. PUBVET, Londrina, v. 4, no. 37, pp. 1-14.

História da raça Nelore. Zebu para o mundo. Disponível em: http:// www.zebuparaomundo.com/zebu/index.php?option=com content\&task=view\&id=357\&l temid=75. Disponibilizado em: $1 \overline{4}$ jan. 2011.

Holanda, MCR, Barbosa, SBP, Ribeiro, AC \& Santoro, KR 2004, 'Tendências genéticas para crescimento em bovinos Nelore em Pernambuco, Brasil', Archivos de zootecnia, v. 53, no. 202, pp. 185-194.

Instituto Brasileiro De Geografia E Estatística (IBGE). 2016. Produção da pecuária municipal. Disponível em: <www.ibge.gov.br/home/ estatistica/economia/ppm/>. Acesso em: 12 fev. 2018.

Instituto Brasileiro De Geografia E Estatística (IBGE). Estatística da produção pecuária. Dezembro de 2016. Disponível em: <www.ibge.gov. $\mathrm{br} /$.../abate-leite-couroovos_201606_publ_completa.pdf?. Acesso em: 07 de jan. 2018.

Kamei, LM, Ribeiro, ELA, Fonseca, NAN, Muniz, CASD, Camiloti, TV, Koritiaki, NA \& Fortaleza, APS 2017, 'Genetic parameters of growth traits in Nellore cattle', Semina: Ciências Agrárias, v. 38, no. 3, pp. 1503-1510.

Lacerda, JJD, Carneiro, PLS, Martins Filho, R \& Malhado, CHM 2014, 'Parâmetros e tendências genéticas para características de crescimento em bovinos da raça Nelore no Estado da Bahia utilizando Inferência Bayesiana', Revista Brasileira de Saúde e Produção Animal, v. 15, no. 1, pp. 10-19.

Lira, T, Rosa, EM \& Garnero AV 2008, 'Parâmetros genéticos de características produtivas e reprodutivas em zebuínos de corte (REVISÃO) ', Ciência Animal Brasileira, v. 9, no. 1, pp. 1-22.

Lôbo, RNB, Martins, JAM, Malhado, CHM, Martins Filho, R \& Moura, AAA 2002, 'Correlações entre características de crescimento, abate e medidas corporais em tourinhos da raça Nelore', Revista Ciência Agronômica, Fortaleza, v. 33, no.2, pp. 93-100.

Lôbo, RNB 2004, 'Metodologias aplicadas na estimativa de parâmetros genéticos e avaliações genéticas de caprinos e ovinos', In: Embrapa Caprinos e Ovinos-Artigo em anais de congresso (ALICE). In: SEMANA DA CAPRINOCULTURA E OVINOCULTURA BRASILEIRAS, 4, Sobral. A pesquisa e os avanços tecnológicos contribuindo para o futuro da caprino-ovinocultura brasileira:[anais]. Sobral: Embrapa Caprinos, 13 f. 1 CD-ROM. 
Lopes, FB, Ferreira, JL, Lobo, RB \& Rosa, GJM 2017, 'Bayesian analyses of genetic parameters for growth traits in Nellore cattle raised on pasture', Genetics and molecular research: GMR, v. 16, no. 3, pp. 1-10.

Lopes, FB, Magnabosco, CU, Paulini, F, Silva, MC, Miyagi, ES \& Lôbo, RB 2013, 'Genetic analysis of growth traits in polled Nellore cattle raised on pasture in tropical region using Bayesian approaches', PLoS One, v. 8, no. 9, p. 1-6.

Marques, EG, Magnabosco, CU, Lopes, FB \& Silva, MC 2013, 'Estimativas de parâmetros genéticos de características de crescimento, carcaça e perímetro escrotal de animais da raça Nelore avaliados em provas de ganho em peso em confinamento', Bioscience Journal, v. 29, no. 1, pp. 159-167.

Martínez-González, JC, García-Esquivel, FJ, Parra-Bracamonte, GM, Castillo-Juárez, H \& Cienfuegos-Rivas, EG 2010, 'Genetic parameters for growth traits in Mexican Nellore cattle', Tropical animal health and production, v. 42, no. 5, pp. 887-892.

Nepomuceno, LL, Andrade, RJ, Lopes, FB, Lira, TS, Vieira, LF, Santos, GCJ \& Ferreira, JL 2017, 'Genetic associations between scrotal circumference and productive and reproductive traits in herd Nellore raised in northern Tocantins State, Brazil', Revista Acadêmica: Ciência Animal, v. 10 , no. 3, pp. 253-261.

Nogueira, DA. Sáfadi, T, Bearzoti, E \& Bueno-Filho, JSS 2003, ‘Analise clássica e bayesiana de um modelo misto aplicado ao melhoramento animal: uma ilustração', Ciência e Agrotecnologia, Lavras, v. 27, pp. 1614-1624.

Pedrosa, VB, Eler, JP, Ferraz, JBS \& Pinto, LFB 2014, 'Utilização de modelos unicaracterística e multicaracterística na estimação de parâmetros genéticos na raça Nelore', Arquivo Brasileiro de Medicina Veterinária e Zootecnia, v. 66, no. 6, pp. 1802-1812.

Pereira, C, Faria, CU \& Lôbo, R 2017, 'The importance of quality information to predict breeding values for growth traits in Nelore cattle', Arquivo Brasileiro de Medicina Veterinária e Zootecnia, v. 69, no. 2, pp. 465-473.

Pinto, C, Ramos, APS, Ozorio, LM \& Brandão, LET 2015, 'Incerteza e Flexibilidade na Pecuária de Corte Brasileira: o Valor da Opção de Confinamento', BBR - Brazilian Business Review, v. 12, no. 6, pp. 102-123.

Resende, MDV, Prates, DF, Yamada, CK \& Jesus, AD 1996, 'Estimação de componentes de variância e predição de valores genéticos pelo método da máxima verossimilhança restrita (REML) e melhor predição linear não viciada (BLUP) em Pinus', Boletim de Pesquisa Florestal, v. 32 , no. 33, pp. 18-45.

Rodrigues, AE, Marques, JRF, Araújo, CV, Camaro Junior, RNC \& Dias, LNS 2010, 'Estimação de parâmetros genéticos para características produtivas em búfalos na Amazônia Oriental', Arquivo Brasileiro de Medicina Veterinária e Zootecnia, v.62, no.3, pp.712-717.

Santana, JG \& Josahkian, AL 201 1, `Efeitos da endogamia em zebuínos com ênfase na raça nelore', Cadernos de Pós-Graduação da FAZU, v. 1, pp. 1-8.

Santos, GCJ, Lopes, FB, Marques, EG, Silva, MC, Cavalcante, TV e Ferreira, JL 2012, 'Tendência genética para pesos padronizados aos 205, 365 e 550 dias de idade de bovinos nelore da região Norte do Brasil', Acta Scientiarum Animal Sciences, v. 34, no. 1, pp. 97-101, 2012.

Santos, MC, Belik, W, Zen, S \& Almeida, LH 2014, 'A rentabilidade da pecuária de corte no Brasil', Segurança Alimentar e Nutricional, v. 21 , no. 2, pp. 505-517.
Santos, NPS, Figueiredo Filho, LAS, Sarmento, JLR, Martins Filho, R, Biagiotti, D \& Neto, AAR 2013, 'Estimação de parâmetros genéticos de pesos em diferentes idades em bovinos da raça Nelore criados no Meio Norte do Brasil usando Amostragem de Gibbs', Acta Tecnológica, v. 7, no. 2, pp. 1-7.

Silva, JAIV, Marcelo, ET, Ribeiro, CB, Maiorano, AM, Curi, RA, Oliveira, HN \& Mota, MDS 2012, 'Análise genética de características de crescimento e perímetro escrotal em bovinos da raça Brangus', Pesquisa Agropecuária Brasileira, v. 47, no. 8, pp. 1166-1173.

Silva, JAI, Ribeiro, CB, Maiorano, AM, Hadlich, JC, Curi, RA, Oliveira, HN\&Meirelles, PRL 2015, 'Influência de fatores ambientais sobre pesos pré-desmama de bovinos cruzados Aberdeen Angus x Nelore', Revista Brasileira de Saúde e Produção Animal, v. 16, no. 2, pp. 278-289.

Sousa Júnior, SC, Araújo Neto, FR, Oliveira, HND, Santos, D, Albuquerque, LGD, Rufino, JEDS \& Martins Filho, R. 2011 , 'Estimação bayesiana de componentes de (co) variância de características ponderais na raça Tabapuã', Revista Brasileira de Saúde e Produção Animal, v.12, no.2, pp.350-358.

Sousa, JER, Sarmento, JL, Sousa, WH, Souza, MS \& Fridrich, AB 2009, 'Estimativas de componentes de covariância e parâmetros genéticos de pesos corporais em caprinos Anglo-Nubiano', Revista Brasileira de Ciências Agrárias, v. 4, no. 2, pp. 211-216.

Souza, LA, Caires, DN, Carneiro, PLS, Malhado, CHM \& Martins Filho, R. 2010, 'Curvas de crescimento em bovinos da raça Indubrasil criados no Estado do Sergipe', Revista Ciência Agronômica, v. 41, no. 4, pp. 671-676.

Valente, BD, Silva, MA, Silva, LOC, Bergmann, JAG, Pereira, JCC, Fridrich, AB \& Corrêa, GSS 2008, 'Covariance structure of body weight in function of age for Nellore animals from southeast and center west of Brazil', Arquivo Brasileiro de Medicina Veterinária e Zootecnia, v. 60 , no. 2, pp. 389-400.

Weber, T. Rorato, PRN, Lopes, JS, Comin, JG, Dornelles, MDA \& Araújo, RD 2009, 'Parâmetros genéticos e tendências genéticas e fenotípicas para características produtivas e de conformação na fase pré-desmama em uma população da raça Aberdeen Angus', Revista Brasileira de Zootecnia, v. 38, no. 5, pp. 832-842.

Yokoo, MJI, Rosa, GJM, Albuquerque, LG, Magnabosco, CU \& Cardoso, FFO 2013, 'O uso da estatística Bayesiana no melhoramento genético animal: uma breve explicação', Scientia Agraria Paranaensis, v. 12, no. 4, pp. 247-257. 